\title{
Razonabilidad y responsabilidad: a propósito de la teoría del derecho contractual del profesor Martín Hevia
}

\author{
Íñigo de la Maza Gazmuri*
}

Resumen: En este trabajo me dedicaré a la idea del profesor Hevia según la cual las relaciones contractuales se basan en "términos de interacción justos", fundados en la reciprocidad y en la injusticia de la imposición unilateral de las condiciones de contratación. Expondré las dificultades que giran en torno a dicha expresión y a sus supuestos, y analizaré el problema que suscita para cierto tipo de contratos (por ejemplo, los contratos de adhesión) la adopción de las tesis que sostiene Hevia. Además, cuestionaré algunas ideas de Hevia con respecto al objeto de los contratos y a la racionalidad instrumental ínsita en la actividad contractual.

Palabras clave: derecho contractual, reciprocidad, razonabilidad.

\begin{abstract}
In this paper, I will focus on the professor Hevia's idea according to which the contractual relations are based on "fair interaction terms", founded on reciprocity and unfairness of the unilateral imposition of the terms of contract. I will expose the difficulties that this expression brings, and I will analyze the problem that the adoption of Hevia's thesis generates for certain type of contracts (for example, standard-form contracts). Besides, I will question some of Hevia's ideas concerning to the object of the contracts and instrumental rationality inherent in contractual activity.
\end{abstract}

Key words: contractual law, reciprocity, reasonableness.

* Abogado; Doctor en Derecho. Profesor Investigador Facultad de Derecho de la Universidad Diego Portales. Profesor de Derecho Civil. Dirección postal: República 105, Santiago de Chile.

Correo electrónico: inigo.delamaza@udp.cl. 


\section{I}

El profesor Martín Hevia nos ofrece una teoría del derecho contractual. Se trata de una empresa poco frecuente en el mundo del civil law, generalmente, más preocupado de explicar el derecho de contratos que de justificarlo. Y esta es una razón para agradecérselo.

El profesor Lucas Grossman me ha pedido que comente esa teoría, tal y como está planteada en un artículo de Hevia que aparece en esta misma publicación ${ }^{1}$. Aprovecho, entonces, para agradecer a Lucas la gentileza de considerarme.

En fin, aunque resultará evidente a partir de mi comentario, comienzo aclarando que la disciplina en que me he especializado es Derecho Civil, particularmente daños y obligaciones. Y me he especializado, digámoslo así, desde una perspectiva doctrinaria ${ }^{2}$. Por lo mismo es que desde ese lugar es donde me siento más cómodo para cumplir con este encargo.

Ante todo, diré que el trabajo del profesor Hevia me resulta atractivo. Considero que su teoría es no solo inteligente, sino además elegante, y eso no es poco. Sospecho, sin embargo, que cuando se le solicita a alguien un comentario bajo estas condiciones no es exactamente para que se limite a reconocer el talento de autor, sino, más bien, para avanzar el diálogo académico o, si se quiere, las ideas; en definitiva, en este caso, para ver si es posible agudizar nuestra comprensión del derecho de contratos.

Dirigiré, entonces, este comentario desde donde pienso (espero no equivocadamente) que puede resultar más útil en esta tarea, es decir, desde mi formación como civilista y, en general, prescindiré - no obstante lo elegante que me parece- del tratamiento de Rawls y su articulación con Kant.

1 Hevia, M., "Razonabilidad y responsabilidad: Rawls, Kant y la teoría del Derecho", Discusiones, XV, 2014, págs. 15-47.

2 Sobre el uso de la expresión "doctrina" puede consultarse Jeztaz, J., $L a$ doctrine, Paris, Dalloz, 2004. 


\section{II}

Forzado a presentar de la forma más sintética mi comentario diría que mi principal observación es que la presentación de la teoría en el artículo que nos interesa se encuentra excesivamente comprimida y esta circunstancia, desde luego, dificulta su lectura.

Creo que, en primer lugar, resulta posible —y útil—descomprimirla respecto del mismo uso de la expresión "teoría". ¿En qué sentido, exactamente, se emplea? La pregunta, según me parece, no constituye una frivolidad analítica, por dos razones. La primera de ellas es que, como muestran $\mathrm{Smith}^{3}$ y Eisenberg ${ }^{4}$, con esa expresión pueden designarse cosas que son diferentes entre sí. La segunda razón — que, en verdad es una consecuencia de la primera- es que únicamente podemos juzgar el éxito de la teoría si sabemos qué es lo que pretende.

Como yo la entiendo, la teoría del profesor Hevia no es ni histórica (no pretende informarnos acerca de cómo y por qué tenemos las reglas que tenemos), ni descriptiva (no pretende hacerse cargo monográficamente del derecho contractual del common law), ni prescriptiva (no aspira a decirnos cómo deberían ser las reglas), sino interpretativa, en el sentido que da Smith ${ }^{5}$ a esa expresión, en la medida en que nos sugiere una idea a partir de la cual resulta posible agudizar nuestra comprensión del derecho de contratos, pues nos revela un orden inteligible que subyace al diseño de sus normas.

Pues bien, si esto es correcto, nuestra atención debe dirigirse hacia esa idea. Y esto nos lleva a la primera página del trabajo que toca comentar. Al centrar nuestro interés en el primer párrafo nos encontramos con la siguiente pregunta: ¿Cómo debemos entender el derecho contractual? La respuesta del profesor Hevia es:

3 Smith, S., Contract Theory, Oxford University Press, 2004.

${ }^{4}$ Eisenberg, M., "The Bargain Principle and Its Limits", Harvard Law Review, Vol. 95, 4, 1982, págs. 741-801.

5 Smith, S., op. cit., págs. 4-6. 
... que la justicia requiere que entendamos las reglas contractuales en función de la idea de "términos de interacción justos". La idea subyacente es la de reciprocidad, es decir, la idea de que es injusto que las personas puedan establecer unilateralmente las condiciones de interacción con los demás. Ello, a su vez, requiere concebir a las partes de un contrato como personas libres e iguales que consienten vincularse bajo condiciones que no se imponen unilateralmente.

Por lo tanto, deberemos entender que la idea matriz es "términos de interacción justos". En segundo lugar, no resulta justo que los términos de interacción sean establecidos unilateralmente. Finalmente, a esta idea subyace la asunción de que debemos considerar a las personas como libres e iguales.

Pues bien, probablemente, haríamos bien en realizar un segundo intento de descompresión aquí.

El primer interrogante que deberíamos formularnos es qué es lo que designa la expresión "términos de intercambio justos" pues es en función de esta idea que debemos entender las reglas contractuales.

Descompongamos la expresión en "términos de intercambio" y "justos". ¿Qué son los términos de interacción? A mí, he de confesarlo, esto me resulta un poco enigmático. No porque no pueda darle sentido, sino más bien porque no estoy seguro acerca de qué sentido darle.

Me surgen dudas acerca de a qué corresponden exactamente los "términos de interacción" y si significan lo mismo o algo diverso de las "condiciones de interacción". Teniendo en cuenta que lo que el profesor Hevia nos pide que entendamos, en función de la idea de términos de intercambio justos, son las reglas contractuales, supongo que habrá que entender que "reglas contractuales", "términos de interacción" y "condiciones de interacción" vienen a ser lo mismo. Sin embargo, utilizar tres expresiones distintas complica las cosas. Por otra parte, si significan cuestiones distintas (y es mi torpeza la que me lleva a confundirlas), probablemente, sería bueno explicarlo.

Ahora bien, supongamos que son lo mismo, lo que tenemos, en principio es que la justicia exige que los términos de intercambios sean justos. Para aligerar esta frase de un cierto sabor circular, debemos 
detenernos en la idea subyacente de reciprocidad. Y tendremos que entender que esa idea de reciprocidad exige considerar que es injusto que las personas puedan establecer unilateralmente las condiciones de interacción con los demás.

De esta manera, podremos afirmar que los términos de interacción justos son aquellos que respetan la reciprocidad y esto significa que las condiciones de interacción no hayan sido impuestas unilateralmente.

Llegados a este punto convendrá advertir que la teoría propuesta puede querer significar dos cosas distintas. La primera es que los términos de interacción son justos en la medida en que no hayan sido impuestos unilateralmente. La segunda es que un requisito que debe satisfacerse para que los términos de interacción sean considerados justos es que no hayan sido impuestos unilateralmente.

Antes de explicar cada una de estas cosas, creo conveniente advertir que, cualquiera sea la decisión que tomemos, va a resultar necesario explicitar qué queremos decir con "impuesto unilateralmente". Creo que esta idea se aclarará al tratar las dos cosas que puede querer significar la teoría.

Consideremos, en primer lugar la teoría como justo igual no impuesto unilateralmente. Nuestra distinción sería la siguiente: los términos del contrato fueron impuestos al otro contratante o los aceptó. Si le fueron impuestos, son injustos; si los aceptó, son justos. Pues bien, hay algo obvio en la importancia que tiene la aceptación de los términos por las partes de un contrato. Lo que no es tan obvio es la relación que existe entre la aceptación del contrato y la justicia de sus términos.

Comencemos con lo obvio. En general, tanto tratándose del common law como del civil law, el contrato es algo que requiere de la voluntad de las partes. En el lenguaje del derecho civil diríamos que si no hay voluntad de una de las partes, según se quiera, el contrato es inexistente o nulo. De esta manera, según me parece, sobre la imposición unilateral el problema, no se trata - al abrigo del derecho de los contratos- si es justo o no; sino, más bien, si hay contrato o no.

Pero, quizás lo que el profesor Hevia quiere decir es algo más sutil. Ya no es la cuestión suficientemente obvia según la cual el contrato 
necesita de las voluntades de quienes quedan obligados por él, sino que, más bien, se trata de aquello a lo que las partes quedan obligadas en el contrato. Y es que, desde un enfoque objetivo del derecho de contratos como el que utiliza el autor ${ }^{6}$, una parte no puede imponer a otra su comprensión de los términos del contrato, sino que el significado de esos términos ha de ser el que le hubieran dado personas razonables. Esta lectura es, desde luego, más sugerente que la anterior. Sin embargo, mi impresión es que conlleva otros problemas.

El enfoque objetivo que nos propone el profesor Hevia nos presta utilidad - y mucha - cuando se suscitan dudas respecto de la debida inteligencia del contrato ya sea respecto del error o de cuestiones relativas a la interpretación cuando existen vacíos. Sin embargo, no parece prestar demasiada utilidad cuando no se suscitan estos problemas. Por lo mismo, si ha existido voluntad y si no existen cuestiones relativas al error o dudas frente a la interpretación, deberemos aceptar que los términos del contrato son justos, y esto tiene que ver con que las personas que consintieron en vincularse deben ser tratadas como libres e iguales ${ }^{7}$. Se trata, entonces, de una vieja idea que expresó con elegancia Alfred Fouillée: "Qui dit contractuel, dit juste".

Ahora bien, aquí me gustaría apropiarme de una crítica de Sandel a Rawls y es que el haber acordado un contrato no garantiza la justicia de sus términos ${ }^{8}$. Particularmente si el contrato no es hipotético, sino real.

Creo que esto es particularmente importante tratándose de una enorme parcela del derecho de contratos, aquella referida a las relaciones de consumo. Si no me equivoco, tratándose de la contratación en materia de consumo, la frase ya no es "quien dice contractual dice justo", sino, al revés: "quien dice justo dice contractual". Y esto tiene que ver con que la métrica de la justicia no tiene que ver con el acuerdo, sino que se encuentra fuera del acuerdo.

${ }^{6}$ Hevia, M., op. cit., pág. 41.

7 Ibídem, pág. 17.

8 Sandel, M., Justicia ¿Hacemos lo que debemos?, Debate, 2011, pág. 167. Debo esta idea a la profesora Beatriz Arriagada y le agradezco su gentileza de haberla compartido conmigo. 
¿Por qué sucede esto? La respuesta está asociada a la racionalidad instrumental, o si se quiere, más bien, a la falta de racionalidad instrumental ${ }^{9}$. Esto resulta particularmente obvio en lo que se denomina contratos por adhesión (standard form contracts), frente a los cuales (1) solemos asumir que los consumidores no se comportarán racionalmente y (2) por lo mismo, no utilizamos el acuerdo como métrica de la justicia de los términos, sino que nos servimos de una métrica externa ${ }^{10}$.

Pues bien, esta situación nos deja con un problema pues encontramos una parcela realmente importante del derecho de contratos (la correspondiente a los contratos por adhesión) en que el acuerdo y la justicia de los términos se encuentran disociados. Y esta disociación se explica porque falla uno de los poderes morales propios de la concepción normativa de la persona sustentada por el profesor Hevia $^{11}$ : la racionalidad instrumental.

¿Qué hacer frente a esa parcela de la actividad contractual? Las posibilidades, creo, son dos. La primera de ellas consiste en señalar que la contratación con los consumidores bajo esas peculiares condiciones de asimetrías informativas y deficiente racionalidad que suelen imponer los contratos por adhesión no forma parte del derecho de contratos en el sentido en que lo entiende esta teoría. Sin embargo, esa respuesta tiene dos problemas. El primero de ellos es que, atendida la enorme importancia de la contratación con consumidores, no es muy evidente que una teoría describa adecuadamente el estado de la cuestión si simplemente prescinde de esta área. Creo que, de esa manera, arriesgaría uno de los requisitos que $\mathrm{Smith}^{12}$ ha planteado

9 Sobre este punto la literatura es particularmente copiosa, a guisa de ejemplo puede consultarse Hanson, J. y Kysar, D., "Taking Behavioralism Seriously: The Problem of Market Manipulation, New York University Law Review, vol. 74, 1999, págs. 630-749; Trebilock (1993, 147-164) y Eisenberg (1995).

${ }^{10}$ De la Maza, I., "Contratos por adhesión y cláusulas abusivas: ¿por qué el Estado y no solamente el mercado?". Revista Chilena de Derecho Privado, Fernando Fueyo Laneri No 1, 2003, págs. 109-148, y De la Maza, I., "E1 control de las cláusulas abusivas y la letra g", Revista Chilena de Derecho Privado, Fernando Fueyo Laneri 3, 2004, págs. 35-68.

${ }^{11}$ Hevia, M., op. cit., págs. 26-27.

${ }^{12}$ Smith, S., op.cit., pág. 10. 
para las teorías de áreas derecho como esta: su adecuación (fit). En sus palabras:

A theory of contract fails the fit criterion if its rejects as an exception or as misclassified so much of contract law that those familiar with the law would not recognize what remains as "contract" law (though such an account might qualify as a good theory of something else).

Ahora bien, se podría cuestionar que la parcela del derecho de contratos que queda excluida sea tan importante como para tornar irreconocible el derecho de los contratos. Sin embargo, aquí viene el segundo problema, si se desea excluir esta área, habría que explicitarlo, y el trabajo, hasta donde llega mi comprensión, no lo hace.

La segunda posibilidad consiste en sostener que, bien pensadas las cosas, la teoría no necesita excluir a los contratos por adhesión porque se trataría de un supuesto en el que, en verdad, las condiciones se han impuesto unilateralmente pues, en realidad, el consumidor no tenía forma de conocer realmente el contenido del contrato.

Esta me parece una buena salida, sin embargo, el problema que tiene - este es el segundo problema de la respuesta - al interior del trabajo es que carecemos de una explicación acerca de qué significa "imponer unilateralmente".

Consideremos ahora la segunda posibilidad, y es que un requisito que debe satisfacerse para que los términos de interacción sean considerados justos es que no hayan sido impuestos unilateralmente. Sin embargo, tratándose de un requisito necesario, no es suficiente, pues, como ha quedado dicho, la métrica de la justicia podría resultar externa al acuerdo.

Esta segunda posibilidad nos 1levaría a lo que Eisenberg ${ }^{13}$ ha denominado una "teoría multivalórica del derecho de contratos". En sus palabras:

${ }^{13}$ Eisenberg, M., "The Theory of Contracts", en Benson (ed.), The Theory of Contract Law. New Essays, Cambridge University Press, 2001, pág. 240. 
In contract law, as in life all meritorious values must be taken into account, even if those values may sometimes conflict, and even at the expense of determinacy. Single-value, metric theories of the best content law must inevitably fail precisely because they deny the complexity of life ${ }^{14}$.

El punto, sin embargo, es que la teoría del profesor Hevia no nos ofrece otros valores que el respeto del acuerdo de personas libres e iguales $\mathrm{o}$, si se quiere, racionales y razonables. Y esto puede ser problemático porque nos deja a ciegas respecto de ciertos límites y respecto del tipo de colaboración que las personas pueden regular a través de un contrato. Para hacer el punto más general, una teoría multivalórica considera que el acuerdo es importante, pero no es una condición suficiente del valor del contrato ${ }^{15}$.

\section{III}

Quisiera ahora dirigir mi atención a otro punto, a la pregunta acerca de qué puede ser objeto de un contrato ${ }^{16}$. Y aquí quiero discutir dos cuestiones. Ambas surgen de la respuesta del profesor Hevia ${ }^{17}$ a la pregunta recién planteada: "con la excepción de las personas independientes, todo puede ser objeto de un derecho adquirido".

Me detengo primero en "con la excepción de las personas independientes, todo (...)". Sin embargo, esa respuesta no parece correcta. No sin una importante cualificación al menos. Desde luego, de acuerdo con la teoría del profesor Hevia, podemos descontar aquellos objetos de un contrato que dañen derechos o intereses protegidos de terceros, pues de esta manera se les estarían imponiendo los términos del contrato a terceros sin que los aceptaran. Se violaría, de esta manera,

${ }^{14}$ Ibídem, pág. 241.

${ }^{15}$ Gordley, J., "Contract Law in the Aristotelian Tradition", en P Benson (ed.) The Theory of Contract Law. New Essays, Cambridge University Press, 2001, pág. 283.

${ }^{16}$ Hevia, M., op. cit., pág. 33.

${ }^{17}$ Ídem. 
la exigencia kantiana de tratar a las personas como libres e iguales. Pero sucede que las limitaciones que se imponen a los contratos respecto de aquello que pueden contratar exceden con generosidad los derechos de terceros. Así, por ejemplo, pasa con ciertas drogas o con los casos de lesión enorme en el derecho continental o los límites a los liquidated damages ${ }^{18}$ y los límites frente al pacto de renuncia al cumplimiento específico en el common law ${ }^{19}$.

Por lo tanto, la respuesta correcta sería más bien que puede ser objeto del contrato todo lo que el Derecho permite. El punto, sin embargo, es que esta cualificación acerca de lo que puede ser objeto de un contrato nos fuerza a pensar qué es lo que el Derecho prohíbe y por qué lo prohíbe. Y ninguna de estas preguntas llega, si quiera, a formularse en el trabajo ${ }^{20}$.

A continuación quiero prestar atención a lo segundo: "puede ser objeto de un derecho adquirido". En la lógica del derecho continental los contratos constituyen fuentes de obligaciones, es decir, su estructura suele correlacionar un crédito con un deber de prestación. Esta estructura es perfectamente consistente con la idea de Kant según la cual aquello que adquiero no es una cosa externa, sino la promesa del otro contratante

El problema, sin embargo, es que no necesariamente sucede así. Por lo pronto, no sucede de esta manera en lo que podríamos denominar contratos reales, en que el negocio se perfecciona por la entrega de la cosa, de manera que ningún crédito ingresa al patrimonio de aquella parte que se obliga a restituir la cosa. Tampoco sucede, si se considera el derecho francés, en el caso del contrato de compraventa pues, como resulta bien sabido, sus efectos son reales.

De esta manera, el punto es que del hecho de que, generalmente, los contratos transfieran créditos no se sigue que necesariamente lo hagan, pues, como acabamos de ver, existen casos en que lo que se

${ }^{18}$ Farnsworth, A., Contracts, Aspen, New York, 1999, pág. 844.

19 Treitel, G., The Law of Contract, 11 $1^{\text {th }}$ ed., Sweet \& Maxwell, 1999, pág. 995.

${ }^{20}$ Sobre el tema creo que el trabajo de Radin resulta particularmente interesante, en Radin, M., Contested Commodities, Harvard University Press, 1996. 
transfiere son cosas distintas a los créditos. Como sea que fuere, se trata de un punto menor, la verdad es que la regla general (y extremadamente) es que los contratos transfieran créditos.

\section{IV}

La siguiente cuestión que quisiera considerar brevemente se refiere a las acciones en caso de incumplimiento. La pegunta que se formula el texto es cuáles son las acciones en caso de incumplimiento ${ }^{21}$.

Ante todo, la respuesta que nos entrega el texto no es completamente leal con la pregunta porque, si bien es cierto que en el common $l_{a} w^{22}$ la indemnización por las expectativas frustradas es la acción estándar, no es la única. Sin embargo, también es verdad que los equitable remedies resultan más bien excepcionales, que la restitution obedece más a la lógica del enriquecimiento sin causa y que él único al que realmente se presta atención en los textos de contratos es el cumplimiento específico (specific performance).

Ahora bien, al concluir su tratamiento de las acciones el profesor Hevia señala ${ }^{23}$ :

En resumen, la indemnización por los daños causados por la frustración de expectativas redime el derecho del acreedor al cumplimiento de la obligación porque le da al acreedor los medios a los que este tenía derecho en virtud del contrato.

El punto, sin embargo, es que no necesariamente lo redime y, quizás, sería conveniente advertir que, cuando no lo redime, bajo ciertos requisitos, se puede solicitar el cumplimiento específico de lo prometido $^{24}$.

${ }^{21}$ Hevia, M., op. cit., pág. 36.

${ }^{22}$ La cuestión es más problemática en el civil law donde resultaría inexplicable que no se considerara la resolución, la suspensión del cumplimiento ni la adecuación del precio.

${ }^{23}$ Hevia, M., op. cit., pág. 40.

${ }^{24}$ Treitel, G., op cit., págs. 949-958. 


\section{V}

Mi último comentario se refiere a la formación del consentimiento. Yo creo que el profesor Hevia lleva razón cuando defiende un estándar objetivo para determinar el contenido del contrato ${ }^{25}$. Tengo mis dudas, sin embargo, respecto del uso que hace de las ideas de Holmes.

Será conveniente transcribir el párrafo que utiliza:

Si... un hombre nace torpe y apresurado, si siempre tiene accidentes y se lastima o lastima a sus vecinos, no hay duda de que sus defectos congénitos estarán permitidos en los tribunales del Cielo, pero sus golpes y caídas no serán menos problemáticos para sus vecinos que si surgen de su negligencia culpable. Sus vecinos le exigen en consecuencia, a su propio riesgo, que se acerque a su estándar, y los tribunales que los vecinos establecen se oponen a tomar en cuenta la ecuación personal de riesgo de dicho individuo.

Comencemos considerando por qué, en mi opinión, Hevia lleva razón. Al señalar que el derecho se preocupa de la apariencia objetiva en los contratos, Treitel ${ }^{26}$ nos señala que la razón que justifica esta opción es la protección de la seguridad jurídica, pues:

Considerable uncertainty would result if $\mathrm{A}$, after inducing $\mathrm{B}$ reasonably to believe that he (A) had agreed to certain terms, could then escape liability merely by showing that he had no "real intention" to enter that agreement.

La función del test objetivo no es, entonces, digámoslo así, responsabilizar a una parte por su torpeza, sino proteger a quien confió en la apariencia creada por la persona torpe.

De esta manera, estoy de acuerdo con el profesor Hevia, simplemente creo que, al considerar las ideas de Treitel, podemos añadir un matiz. Señala Hevia ${ }^{27}$ que 'los pensamientos 'internos' de las partes

5 Hevia, M., op. cit., pág. 41.

26 Treitel, G., op. cit., pág. 1.

27 Hevia, M., op. cit., pág. 41. 
no son definitivos para determinar la existencia o el contenido de un contrato". Esto, me parece, es correcto. El matiz, sin embargo, es que pueden serlo. Y pueden serlo porque, hemos de recordar, la función del test objetivo no es sancionar a quien se comporta de manera negligente, sino proteger a quien confía en la declaración.

Imaginemos un ejemplo que ilustre lo que quiero decir. Con su conducta torpe A puede crear una falsa apariencia — esta sería la apariencia objetiva - de querer celebrar un contrato, imaginemos que, por alguna razón $B$ sabe que esa apariencia objetiva no se compadece con la realidad, que el pensamiento interno de $A$ es que no desea celebrar el contrato. Imaginemos, sin embargo, que B manifiesta su aceptación respecto de esa apariencia objetiva, ¿Se ha formado el contrato? La respuesta es, desde luego, que no. El pensamiento interno de A resultará definitivo para determinar la inexistencia del contrato pues preferimos proteger a quien se comporta negligentemente que a quien busca aprovecharse de esa negligencia.

Como digo, sin embargo, se trata de un matiz con el cual, estoy seguro, el profesor Hevia estaría de acuerdo conmigo. Por lo mismo, no tiene sentido insistir.

Bajo la formación del consentimiento me interesa, aún, hacer otra prevención que se refiere al uso de las ideas de Holmes por parte del profesor Hevia. Comenzaré por la cita de Holmes de la que se sirve el autor ${ }^{28}$ :

Si... un hombre nace torpe y apresurado, si siempre tiene accidentes y se lastima o lastima a sus vecinos, no hay duda de que sus defectos congénitos estarán permitidos en los tribunales del Cielo, pero sus golpes y caídas no serán menos problemáticos para sus vecinos que si surgen de su negligencia culpable. Sus vecinos le exigen en consecuencia, a su propio riesgo, que se acerque a su estándar, y los tribunales que los vecinos establecen se oponen a tomar en cuenta la ecuación personal de riesgo de dicho individuo. 
Ahora bien, Holmes parece estar en lo correcto cuando afirma que, tratándose de responsabilidad extracontractual, los tribunales no toman en cuenta la ecuación personal de riesgo de la persona, digamos, torpe. Una conclusión semejante se puede defender respecto del mundo del civil law ${ }^{29}$. El punto, sin embargo, es que, al menos a mí, no me resulta tan evidente que en materia contractual no se tome en cuenta la ecuación de riesgo personal de las personas torpes. Por supuesto que se toma respecto de quienes consideramos incapaces, pero esto no es novedad respecto de la responsabilidad extracontractual y no parece ser lo que quiere decir Holmes. Este autor parece referirse a quienes han traspasado un cierto umbral de capacidad que los torna imputables en términos civiles.

En materia contractual, la ecuación de riesgo puede tomarse en cuenta más allá del umbral de la capacidad, en aquellos casos que Eisenberg $^{30}$ ha agrupado bajo el rótulo "doctrine of transactional capacity". En sus palabras:

The doctrine of transactional capacity is not limited to cases where a promisor lacks capacity to understand the value of the performances to be exchanged; it also applies to cases in which the promisor can understand the value of the performance called for but lacks capacity to understand the meaning of contractual provisions governing the parties' rights in the event of nonperformance. This application is most likely to occur in transactions involving form contracts prepared by relatively sophisticated sellers who deal regularly with relatively unsophisticated buyers.

De esta manera, creo que la idea de personas racionales y razonables y el test del consentimiento objetivo constituyen dispositivos muy útiles para enfrentar un número importante de problemas que genera el derecho de contratos, pero deben ser matizadas para enfrentar adecuadamente los problemas que estas mismas ideas pueden generar.

${ }^{29}$ Barros, 2006, pág. 78.

${ }^{30}$ Eisenberg, M., The Bargain... op cit., pág. 771. 
En fin, al concluir con estas palabras, quisiera felicitar al profesor Martín Hevia por este trabajo y el libro del cual proviene. Tengo la amarga sospecha de que buena parte de los comentarios que he hecho se debe no a insuficiencias de la teoría, sino a mi torpeza. Si es así, aprovecho la ocasión para dar las disculpas del caso al autor y expresarle mi confianza en que alguna vez nos reunamos para que me libere del error.

\section{Bibliografía}

De la Maza I., "Contratos por adhesión y cláusulas abusivas: ¿por qué el Estado y no solamente el mercado". Revista Chilena de Derecho Privado, Fernando Fueyo Laneri, 1, 2003, págs. 109-148.

De la Maza I., "El control de las cláusulas abusivas y la letra g)", Revista Chilena de Derecho Privado, Fernando Fueyo Laneri, 3, 2004, págs. 35-68.

Eisenberg M ., "The Theory of Contracts", en P Benson (ed.) The Theory of Contract Law. New Essays, Cambridge University Press, 2001, págs. 206-264.

Eisenberg M.,"The Bargain Principle and Its Limits", Harvard Law Review, 95, 4, 1982, págs.741-801.

Farnsworth, A., Contracts, Aspen, New York, 1999.

Gordley, J., "Contract Law in the Aristotelian Tradition", en P Benson (ed.), The Theory of Contract Law, New Essays, Cambridge University Press, 2001, págs. 265-334.

Hanson, J. y Kysar, D., "Taking Behavioralism Seriously: The Problem of Market Manipulation", New York University Law Review, 74, 1999, págs. 630-749.

Hevia, M., "Razonabilidad y responsabilidad: Rawls, Kant y la teoría del Derecho", Discusiones, XV, 2014, págs. 15-47

Jeztaz, J., La doctrine, Paris, Dalloz, 2004.

Radin, M., Contested Commodities, Harvard University Press, 1996.

Sandel M., Justicia ¿Hacemos lo que debemos?, Debate, 2011.

Smith, S., Contract Theory, Oxford University Press, 2004. 
Íñigo de la Maza Gazmuri

Trebilcock, M., The Limits of Freedom of Contract, Harvard University Press, 1999.

Treitel, G., The Law of Contract, 11 ${ }^{\text {th }}$ ed., Sweet \& Maxwell, 1999. 